\title{
Fundamentos del Comportamiento de Micropilotes Hincados en Suelos Limo-Arenosos de Córdoba
}

\section{Fundamentals of the Behavior of Driven Micropiles in Sandy-Loamy Soils of Córdoba}

Presentación: 6 y 7 de octubre de 2020

\section{Doctorando:}

\section{Rorigo Baldoncini}

Centro de Investigación y Desarrollo en Geotecnia, Estructuras y Fundaciones (CIGEF), Departamento de Ingeniería Civil, Facultad Regional Córdoba, Universidad Tecnológica Nacional - Argentina

rodrigobaldoncini@gmail.com

\section{Director/es:}

\section{Gonzalo Aiassa}

\section{Co-director/es:}

\section{Pedro Arrúa}

\section{Resumen}

La utilización de micropilotes hincados es una alternativa interesante para resolver cimentaciones en proyectos de ingeniería de pequeña y mediana envergadura. Incluso en grandes obras, pueden ser utilizados para estabilizar taludes o como pantalla de sostenimiento en excavaciones de subsuelos. A los fines de plantear el uso en este tipo de soluciones, es necesario contar con procedimientos de diseño validados para suelos locales y la tecnología específica con la que los mismos serán materializados. Se plantea una revisión literaria de procedimientos para determinar capacidad de carga de micropilotes hincados, que contemplen la tecnología constructiva y las características propias del suelo. Se desarrolla un plan experimental de ensayos de campo sobre micropilotes a escala real. Se implementan modelos numéricos para la condición de micropilotes hincados instalados en suelos limosos, limo-arenosos y arenosos. Se desarrollan formulaciones dinámicas con el propósito de estimar la capacidad de carga a partir de los resultados de ensayos de penetración en campo.

Palabras clave: Micropilotes, Hincado, Inyectado.

\begin{abstract}
The use of driven micropiles is an interesting alternative to solve foundations in engineering projects of small and medium scale. Even in large works, they can be used to stabilize slopes or as a support screen in underground excavations. In order to propose the use in this type of solutions, it is necessary to have validated design procedures for local soils and the specific technology with which they will be materialized. In this paper, a literary review of procedures to determine the carrying capacity of driven micropiles, contemplating the construction technology and the characteristics of the soil is proposed. An experimental plan of field tests on micropiles in real scale is developed. Numerical models are implemented for the condition of driven micropiles installed in silty, sandy loamy and sandy soils. Dynamic formulations are developed for the purpose of estimating the load capacity from the results of field penetration tests.
\end{abstract}

Keywords: Micropiles, Driven, Injected. 


\section{Introducción}

La tecnología de los micropilotes fue introducida en Italia en 1.952 por la empresa especialista en fundaciones Fondedile, bajo la dirección técnica del Dr. Fernando Lizzi (Bruce et al., 1997), extendiéndose, dos décadas más tarde, hacia EE.UU. Estos pilotes, menores que los permitidos por los códigos de la época, fueron llamados "pali radice" y fueron concebidos como un método para recalzar edificios históricos y monumentos.

Un micropilote es un pilote de diámetro pequeño, normalmente menor a $300 \mathrm{~mm}$, perforado, armado e inyectado (FHWA, 2005). Existen diferentes variantes en función de la configuración y del método de inyección (Ministerio de Fomento, 2005). El sistema puede aplicarse para reforzar fundaciones existentes, estabilizar taludes y fundar nuevas estructuras.

La práctica típica de construcción incluye las siguientes etapas: perforación del terreno a la profundidad requerida, emplazamiento del refuerzo de acero y finalmente, la inyección a presión de una lechada de cemento. Debido al avance en la implementación de diferentes técnicas y la practicidad del proceso de construcción, su aplicación se ha extendido a diferentes tipos de proyectos. La transferencia de carga se realiza principalmente a través de la fricción desarrollada en el fuste del elemento. Una segunda componente, frecuentemente menor, se transfiere por presión en la punta del micropilote. La capacidad de carga depende de la configuración geométrica, del método de construcción y de las condiciones del suelo. El diseño estructural incluye verificaciones de flexión, corte, tracción, compresión y pandeo.

\section{Desarrollo}

Se ha recopilado una considerable bibliografía y antecedentes de su empleo tanto en el ámbito local (Leoni, 2010, Capdevila et al. 2016, Jacinto et al., 2014) como internacional (Koreck, 1978, Armijo Palacio, 2003, Cadden et al., 2004, Kershaw and Luna, 2014) entre otros, para investigar y estudiar el comportamiento de micropilotes. Se realizó una revisión del estado del conocimiento principalmente, relativos a los temas de pilotes, micropilotes hincados y excavados, metodología constructiva, criterios de diseño, capacidad de carga, estudios experimentales, modelos físicos, interacción suelo-estructura, ensayos de penetración dinámica, formulaciones dinámicas y análisis numérico.

En el campus de la UTN-FRC se cuenta con un laboratorio para pruebas de campo a escala real, en donde se ha diseñado un plan experimental de MHI que se encuentra en proceso de ensayo. Se han contemplado condiciones de inyección y diferentes profundidades de pilotes. Los resultados permitirán ajustar los parámetros de calibración a las condiciones de instalación del método, y al tipo de suelo local. La Figura 1 muestra registro fotográfico durante la construcción del mismo. En la actualidad, en total se ejecutaron 6 MHI, 3 a 4 metros de profundidad y 3 a 8 metros de profundidad. En una segunda etapa se planifica construir a 10 y 12 metros de profundidad. Se desarrollan formulaciones dinámicas con el propósito de estimar la capacidad de carga a partir de los resultados de ensayos de penetración en campo.

Por otra parte, se construyeron 5 (cinco) modelos físicos de MHI para la ejecución de experimentos a escala reducida en laboratorio. En la Figura 2 se indican los parámetros geométricos más relevantes del prototipo de fundación utilizada. Los modelos físicos de MHI se instalaron en dos pasos. Primero se hincó la sección de acero estructural dentro del suelo preparado bajo ciertas condiciones de humedad y moldeado en una probeta. Segundo, se realizó la inyección de la lechada de cemento. La instalación de la armadura tubular se efectuó utilizando una prensa motorizada con una velocidad de avance constante de $1,27 \mathrm{~mm} / \mathrm{seg}$., con retención del valor de carga máxima. Para ello, se ubicó el molde en la prensa, y se acomodaron los accesorios necesarios para la correcta ejecución. Se montó un reloj comparador digital de un rango de medición de $50 \mathrm{~mm}$ y con una sensibilidad de 0,01 $\mathrm{mm}$ para medir desplazamientos verticales de la cabeza del pilote durante el hincado. Se aplicaron gradualmente incrementos de carga y se recolectaron lecturas de cargas para cada valor de penetración de la punta de la armadura tubular, hasta lograr una profundidad de empotramiento de la armadura en la probeta de $105 \mathrm{~mm}$. 


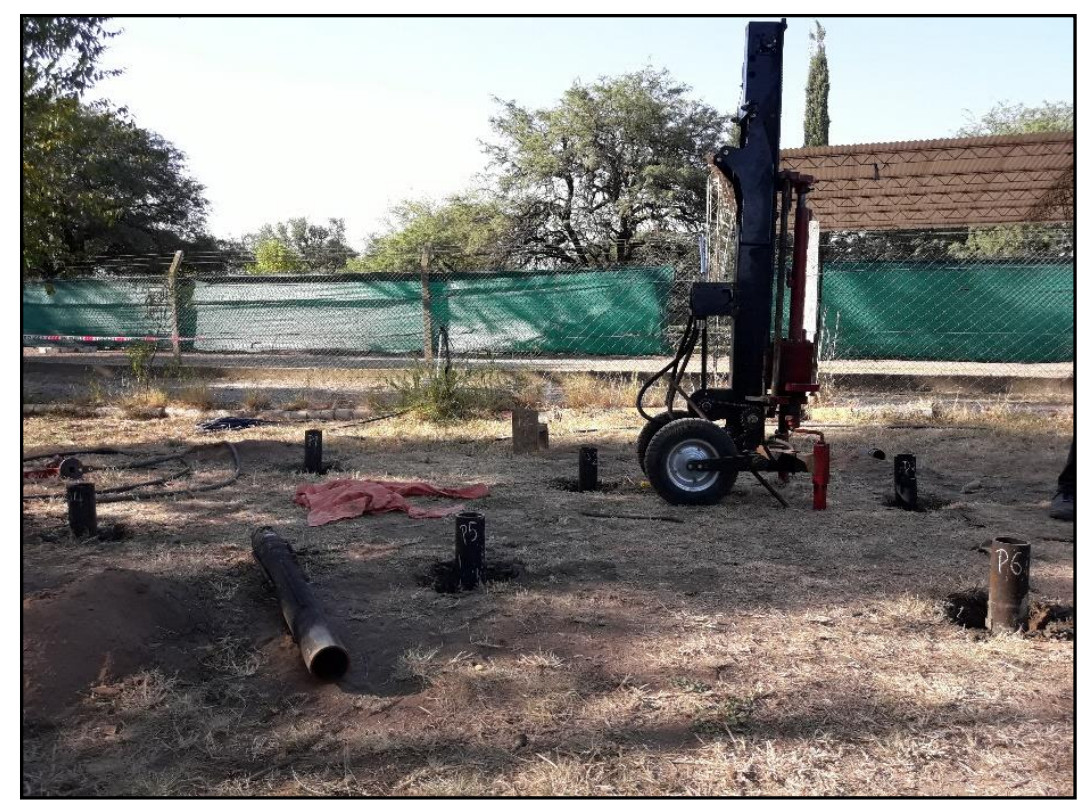

Figura 1: Fase de instalación de MHI en el campus de la UTN-FRC

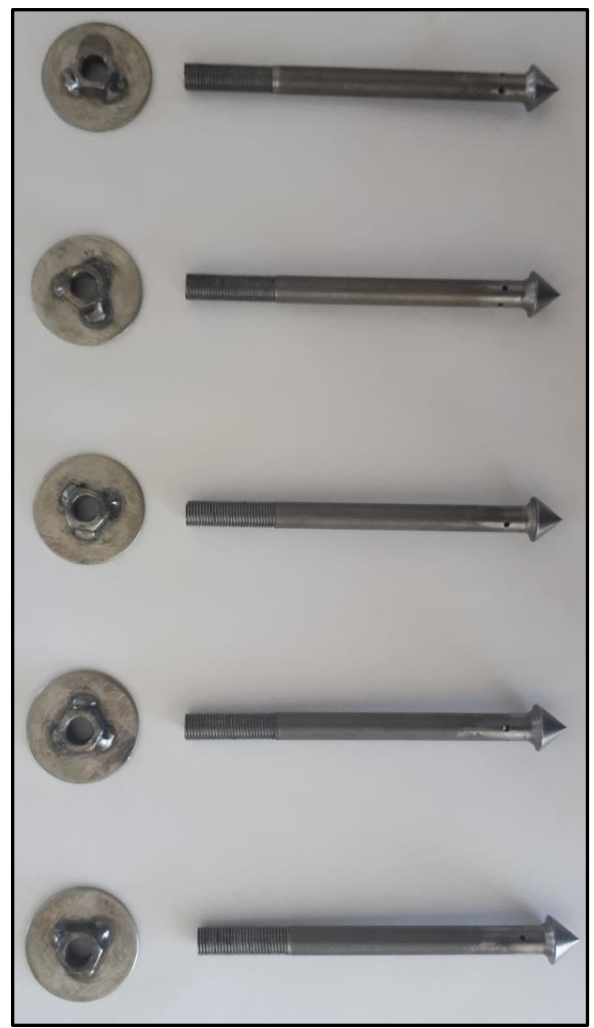

(A)

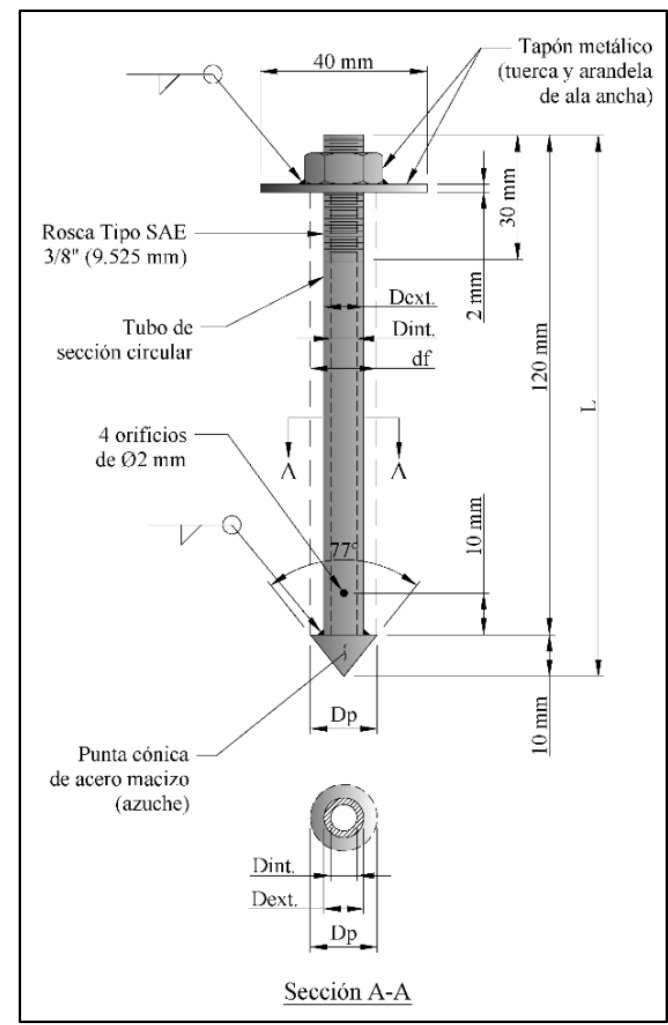

(B)

Figura 2: Modelo físico de la sección de acero del MHI (A) Sección de acero y tapón superior, (B) Geometría

Una vez instalada la armadura tubular en las probetas, se llevaron adelante las pruebas de inyección de lechada de cemento. La propuesta experimental consistió en emplear un tanque cilíndrico de plástico con un diámetro igual a 31 mm y una altura de $117 \mathrm{~mm}$; provisto de un émbolo axial del mismo material y diámetro al del tanque. La Figura 3 muestra una ilustración esquemática de la secuencia constructiva realizada en el laboratorio y la Figura 4 presenta los micropilotes finalmente inyectados y preparados para ensayarlos. 

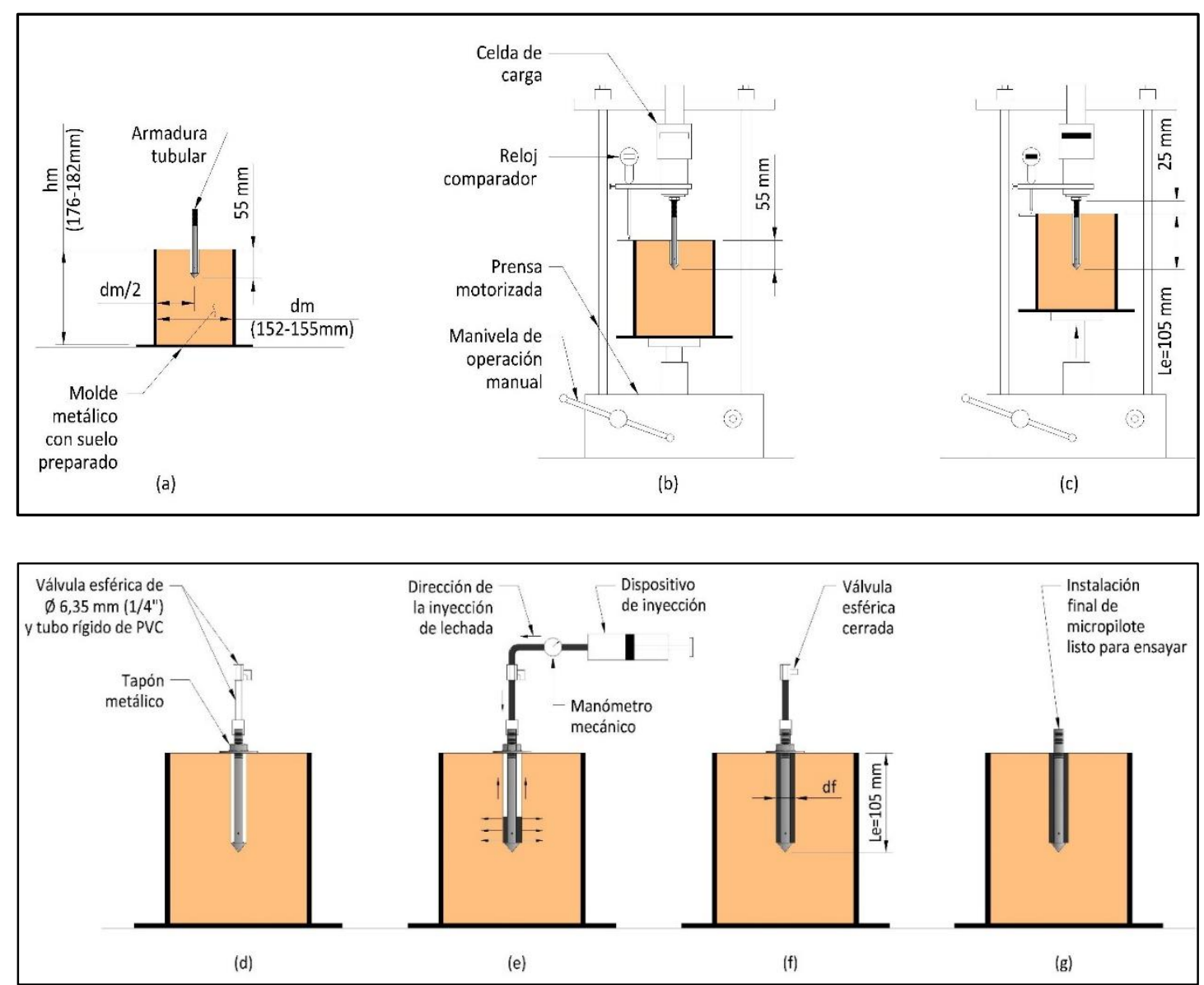

Figura 3: Construcción de modelo físico MHI: Hinca de sección tubular de acero e inyección de lechada de cemento

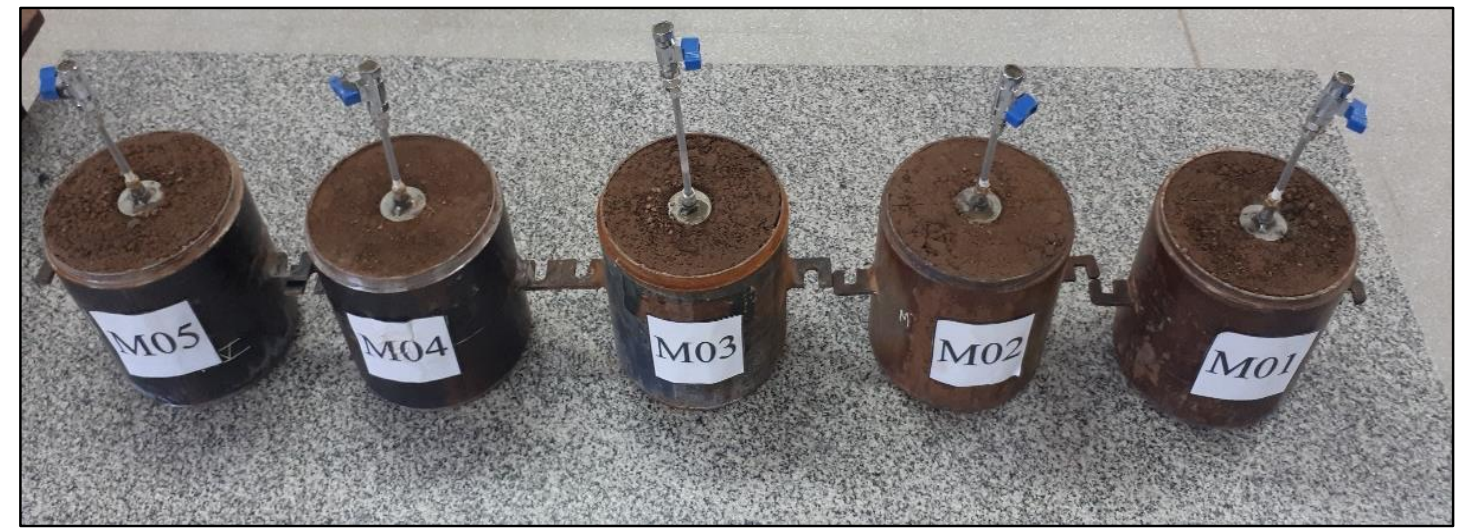

Figura 4: Modelos físicos de MHI: instalados e inyectados

Sobre los modelos físicos de MHI se efectuaron ensayos de carga axial de compresión. Para la ejecución de los ensayos, se empleó un banco de carga de accionamiento mecánico, que permite aplicar esfuerzos de hasta $50 \mathrm{KN}$. Los desplazamientos verticales de la "cabeza" de los MHI, se midieron utilizando un reloj comparador. 


\section{Resultados}

Los resultados obtenidos de los ensayos se relacionaron mediante curvas carga vs. asentamientos (Q-s). De manera ilustrativa, la Figura 5 muestra la curva de comportamiento del micropilote MHI01.

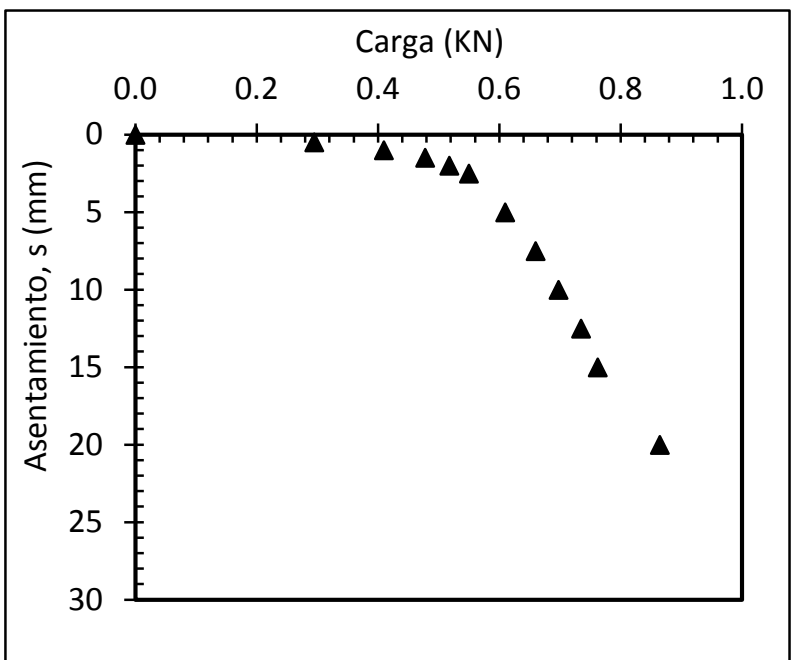

(a) rango de asentamientos: 0-25mm

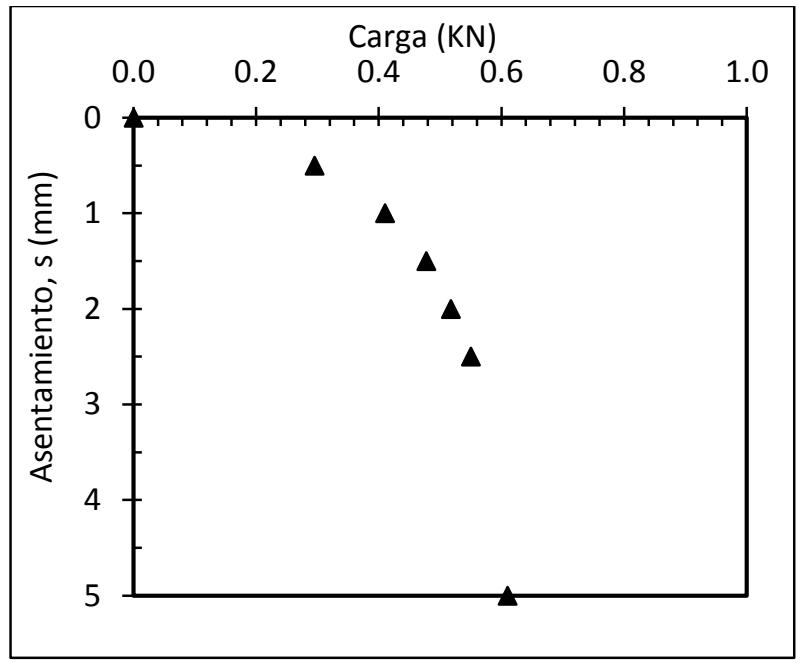

(b) rango de asentamientos: $0-5 \mathrm{~mm}$

Figura 5: Resultados del ensayo carga-asentamiento del micropilote MHI01

Luego, se aplicaron modelos matemáticos de extrapolación y calibración (Fellenius, 1980, Fleming, 1992, Almeida et al., 2018), a las curvas Q-s, basados sobre patrones que se obtienen de dichas curvas, para así calcular la capacidad geotécnica de falla o de rotura. La Figura 6 muestra las curvas de extrapolación del ensayo de carga del micropilote MHI01 y la Tabla 1 resume los valores de capacidades de carga de los micropilotes para cada método propuesto.

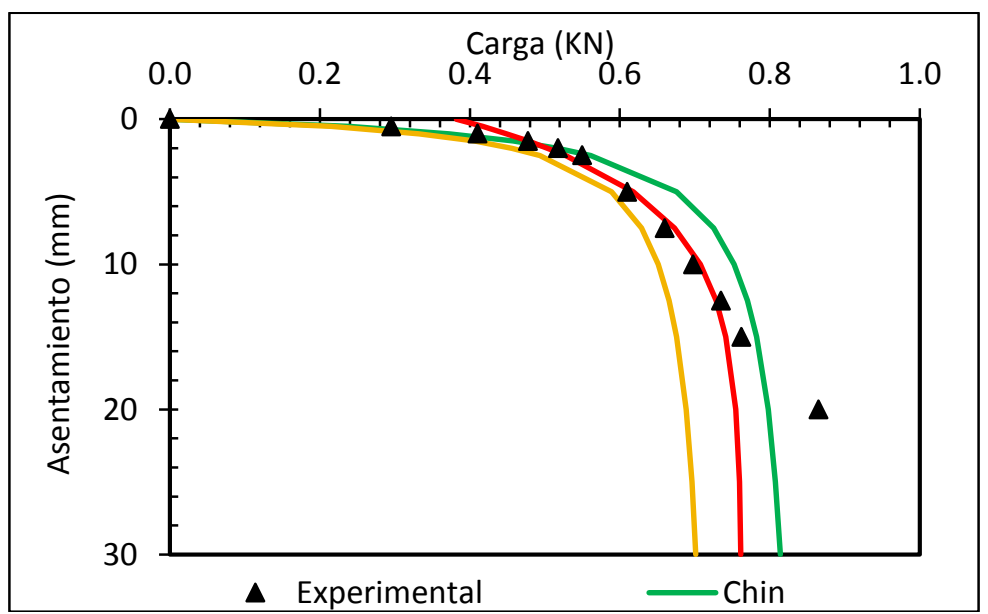

Figura 6: Curvas de extrapolación de MHI01

\begin{tabular}{|c|c|c|c|}
\hline ID & Chin (1971) & Décourt (1996) & Van Der Veen (1953) \\
\hline MHI01 & 0,756 & 0,635 & 0,653 \\
\hline MHI02 & 0,849 & 0,729 & 0,760 \\
\hline MHI03 & 0,681 & 0,663 & 0,687 \\
\hline MHI04 & 2,685 & 2,225 & 2,393 \\
\hline MHI05 & 0,590 & 0,571 & 0,625 \\
\hline
\end{tabular}

Tabla 1: Capacidades de carga total última de MHI, Qútl. (KN) 
También se ha logrado medir la capacidad de carga de fricción y punta de cada MHI, a partir de ensayos de laboratorio sobre modelos físicos de fundación.

Un próximo paso sería la creación y calibración de un modelo de dos dimensiones (2D) de elementos finitos implementando el programa RFEM 5, utilizando los resultados experimentales. Se empleará el modelo para realizar un estudio paramétrico e investigar el comportamiento de micropilotes sometidos a los efectos de una carga vertical de compresión. Los parámetros analizados incluirán las curvas Q-s, la geometría de micropilotes y propiedades del suelo. Basado en éste análisis, se propondrá una metodología para estimar la capacidad de carga de micropilotes para una escala real de campo.

\section{Referencias}

Almeida, A.; Jesswein, M.; Liu, J. (2018). Evaluation of determination methods for ultimate axial capacity of micropiles in Ontario soils. Departament of Civil Engineering. Ryerson University, Ontario, Canada.

Armijo Palacio, G. (2003). Micropilotes con inyecciones de desplazamiento. Jornadas Técnicas SEMSIG-AETESS. CEDEX, Madrid.

Bruce, D. A.; DiMillio, A. F. and Juran, I. (1997). Micropiles: The state of practice. Part I: Characteristics, definitions and classifications. Ground Improvement Journal. 1, pp. 25-35.

Cadden, A.; Gómez, J; Bruce, D. and Armour, T. (2004). Micropiles: Recent advances and future trends. Current Practices and Future Trends in Deep Foundations. ASCE.

Capdevila, J. A.; Francisca, F. M.; y Zanni, E. S. (2016). Patologías estructurales asociadas al ascenso de la napa freática en el sureste de la provincia de Córdoba. XXIII Congreso Argentino de Mecánica de Suelos e Ingeniería Geotécnica. SAIG, Santa Fé.

Federal Highway Administration-National Highway Institute. (2005). Micropiles Design and Construction, Reference Manual. U.S. Departament of Transportation, Washington, D.C..

Fellenius, B. (1980). The analysis of results from routine pile load test. Ground Engineering. Vol. 13, No.6, pp. 19-31.

Fleming, W. (1992). A new method for single pile settlement prediction and analysis. Geotechnique, Vol. 42, No.3, pp. 411-425.

Jacinto, A. C.; Galíndez, E. E. y Dip, O. (2014). Propuesta de recalce del edificio del ex banco Francés de la Provincia del Tucumán. 23o Jornadas Argentinas de Ingeniería Estructural. AIE, Buenos Aires.

Kershaw, K. A. and Luna, R. (2014). Full-scale field testing of micropiles in stiff clay subjected to combined axial and lateral loads. Journal of Geotechnical and Geoenvironmental Engineering. Volume 140, Issue 1, 255 - 261. ASCE.

Koreck, W. (1978). Small diameter bored injection piles. Ground Engineering. Vol.11, No.4, pp.14-20.

Leoni, A. J. (2010). Micropilotes inyectados en suelos duros de la Formación Pampeana. XX Congreso Argentino de Mecánica de Suelos e Ingeniería Geotécnica. SAIG, Mendoza.

Ministerio de Fomento. (2005). Guía para el proyecto y la ejecución de micropilotes en obras de carretera. Dirección Técnica de la Direción General de Carreteras. 